\title{
Long-term follow-up results of unfractionated heparin infusion treatment for submassive pulmonary thromboembolism
}

\author{
Burcu Ozdemir ${ }^{*}$ (), Levent Ozdemir ${ }^{2}$ (D) Bilge Akgündüz ${ }^{3}$ (1)
}

\section{SUMMARY}

OBJECTIVE: Treatment options for submassive pulmonary thromboembolism cases vary depending on the patient's hemodynamic stability, comorbidities, and bleeding risk. The long-term effect of unfractionated heparin treatment on pulmonary hypertension and mortality is unclear. The aim of this study was to investigate the long-term effect of unfractionated heparin treatment on pulmonary thromboembolism. METHODS: This is a cross-sectional study with 22 patients who were diagnosed with submassive pulmonary thromboembolism and followed up at the outpatient clinic between 2016 and 2020 and received unfractionated heparin treatment.

RESULTS: Mean pulmonary artery pressure was $53 \pm 13.6 \mathrm{mmHg}$ during hospital admission and $42.7 \pm 13.4 \mathrm{mmHg}$ at hospital discharge. There was a statistically significant decrease in $\mathrm{D}$-dimer and pulmonary artery pressure levels before and after treatment $(p=0.001)$. At the end of one year, pulmonary artery pressure was considered high in three patients of this study.

CONCLUSION: Our study suggests that unfractionated heparin is safe in the treatment of submassive pulmonary thromboembolism in terms of bleeding risk and reduces pulmonary artery pressure.

KEYWORDS: Pulmonary embolism. Unfractionated heparin. Long-term effect. Pulmonary hypertension.

\section{INTRODUCTION}

Pulmonary thromboembolism (PTE) presents with different clinical characteristics, ranging from asymptomatic cases to those who die within hours due to hemodynamic instability. Due to these clinical differences, treatment approaches also vary. "Risk assessment" is the most critical step in determining the appropriate treatment approach for acute PTE cases. "Risk," as defined herein, is the risk of death associated with the acute PTE. Therefore, distinguishing the patient diagnosed with acute PTE as high risk (massive), intermediate risk (submassive), or low risk (nonmassive) in terms of early mortality can help determine the treatment options and prognosis ${ }^{1}$. Low-molecular-weight heparin (LMWH) and oral anticoagulants are often preferred for the treatment of nonmassive PTE and thrombolytics for massive PTE². Treatment options for submassive PTE cases vary depending on the patient's hemodynamic stability, comorbidities, and bleeding risk ${ }^{3}$. Systemic anticoagulation, LMWH, oral anticoagulants, catheter-directed thrombolysis, half-dose thrombolysis ( $50 \mathrm{mg}$ tPA), and inferior vena cava (IVC) filters are the treatment options for submassive $\mathrm{PTE}^{4,5}$. Unfractionated heparin (UFH) treatment was commonly used in the past for submassive PTE but is less preferred nowadays due to newer treatment options that are easier to follow. Studies investigating the effect of UFH infusion therapy on pulmonary hypertension (PHT) in the long term are insufficient.

\footnotetext{
'Samsun Education and Research Hospital, Chest Disease - Samsun, Turkey.

${ }^{2}$ Dörtyol State Hospital, Chest Disease - Hatay, Turkey.

${ }^{3}$ Eskişehir City Hospital Chest Diseases, Occupational and Occupational Diseases - Eskisehir, Turkey.

*Corresponding author: Levent2408@mynet.com

Conflicts of interest: the authors declare there is no conflicts of interest. Funding: none.

Received on August 10, 2021. Accepted on September 17, 2021.
} 
In this article, we examined the demographic characteristics, symptoms, comorbidities, and risk factors of patients with submassive PTE treated with UFH infusion; the effect of heparin on platelet count and blood count in the early (3-5 days) and late ( 15 days) periods; and the developmental status of chronic thromboembolic PHT at the end of one year.

\section{METHODS}

In our study, data from 22 patients who received UFH infusion therapy after admission to the intensive care unit (ICU) with a diagnosis of submassive PTE and were subsequently followed up in the outpatient clinic for one year were retrospectively analyzed between 2016 and 2020 according to the 2015 Turkish Thoracic Society PTE diagnosis and treatment consensus report.

\section{Study population}

The diagnosis of PTE was made in the emergency department using contrast-enhanced dynamic chest computed tomography (image). Patients with findings consistent with submassive PTE on echocardiography (ECHO) were included in this study. In addition, patients with findings of right ventricular dilatation, paradoxical motion and deviation of the septal wall to the left, moderate or severe hypokinesis suggestive of right ventricular dysfunction, mobile thrombus in the right atrium, PHT, and patent foramen ovale at $\mathrm{ECHO}$, despite normal systemic blood pressure, were considered submassive PTE. Patients' symptoms on admission, concomitant diseases and risk factors, hemoglobin and platelet counts, troponin and D-dimer levels, arterial blood gas values, and ECHO findings on admission to the ICU and on discharge from the hospital were obtained from hospital records. Additionally, follow-up results of ECHO at months one, three, and six and at the end of the first year, as well as D-dimer levels at the end of treatment, were obtained from outpatient records. Subsequently, all collected data were statistically analyzed.

\section{Treatment protocol}

All patients were followed up in the ICU after ECHO was performed in the emergency department. After observing the basal activated partial thromboplastin time (aPTT) values of the patients, a bolus of $80 \mathrm{IU} / \mathrm{kg}$ intravenous (i.v.) followed by a heparin infusion of $18 \mathrm{IU} / \mathrm{kg} / \mathrm{h}$ was started. In the first $24 \mathrm{~h}$, treatment was supplemented with warfarin when the aPTT value reached 45-70. The aPTT level was measured every $6 \mathrm{~h}$ for the first $24 \mathrm{~h}$ and daily after reaching the desired level. If internalized normal ratio
(INR) values of $2-3$ were detected within the $24-\mathrm{h}$ interval, heparin was discontinued and treatment with warfarin was continued. Thus, all patients received warfarin therapy for at least six months.

\section{Statistical analysis}

Statistical analysis was performed using Statistical Package for Social Sciences (SPSS) version 15.0. A homogeneity test was performed on numeric variables. Independent sample $t$-tests were used for numerical parameters. Paired sample $t$-test was used to test whether there was a difference in the mean of numerical variables at the beginning (baseline) and end of treatment. Pearson's correlation analysis was performed for distributed homogeneous variables. Nonhomogeneous variables were analyzed using nonparametric tests. A $\mathrm{p}<0.05$ was considered significant.

\section{RESULTS}

Of the 22 patients followed up and treated with a diagnosis of submassive PTE, 11 were females and 11 were males. The mean age of the cases was $53.5 \pm 20$ years. The most common symptoms on admission were shortness of breath $(\mathrm{n}=22)$, central chest and flank pain $(\mathrm{n}=18)$, palpitations $(\mathrm{n}=16)$, and cough $(n=15)$, while the most common risk factors were immobilization $(n=12)$, deep vein thrombosis $(n=10)$, and orthopedic surgery $(\mathrm{n}=6)($ Table 1$)$.

Blood gas values of patients on admission were as follows: $\mathrm{pH}$ : $7.46 \pm 0.05, \mathrm{PO}_{2}: 66.1 \pm 14.1 \mathrm{mmHg}, \mathrm{PCO}_{2}: 32.4 \pm 5.2 \mathrm{mmHg}$, $\mathrm{HCO}_{3}: 22.1 \pm 3.1 \mathrm{mEq} / \mathrm{L}$, saturation $\mathrm{O}_{2}: 92 \pm 4.7 \%$. Hypoxemia $\left(\mathrm{PO}_{2}<80 \mathrm{mmHg}\right)$ was observed in 18 patients, and hypocapnia $\left(\mathrm{PCO}_{2}<35 \mathrm{mmHg}\right)$ was observed in 15 patients.. D-Dimer levels were $3.67 \pm 2 \mathrm{ng} / \mathrm{mL}$ before treatment and $0.49 \pm 0.59$ $\mathrm{ng} / \mathrm{mL}$ at the end of treatment. D-Dimer levels remained high $(>0.5 \mathrm{ng} / \mathrm{mL}$ ) in two patients at the end of treatment. Of the patients whose $\mathrm{D}$-dimer levels remained high, one had prior cerebrovascular disease and coronavirus 2019 disease, and one had breast carcinoma.

The mean troponin level on admission was $42.3 \pm 50.3 \mathrm{ng} / \mathrm{mL}$. Troponin levels were above normal laboratory values in 12 patients (troponin 0-14 $\mathrm{ng} / \mathrm{mL}$ ).

Mean hemoglobin on hospital admission was $12.2 \pm 2.1$ and $11.2 \pm 1.9 \mathrm{~g} / \mathrm{dL}$ on day three, $11.7 \pm 1.8 \mathrm{~g} / \mathrm{dL}$ on day five, and $11.9 \pm 1.6 \mathrm{~g} / \mathrm{dL}$ on day 15 . The mean platelet count was $291,500 \pm 95,700 / \mathrm{mL}$ on admission, $295,000 \pm 159,100 / \mathrm{mL}$ on day $3,307,300 \pm 158,000 / \mathrm{mL}$ on day 5 , and $341,800 \pm 159,000 / \mathrm{mL}$ on day 15 . No patient experienced a decrease in hemoglobin level or platelet count during the early (on days 3-5) and late (on day 15) phases with heparin. 
Mean pulmonary artery pressure (PAP) was $53 \pm 13.6 \mathrm{mmHg}$ on $\mathrm{ECHO}$ in the emergency department during hospital admission. PAP was found to be $42.7 \pm 13.4 \mathrm{mmHg}$ at hospital discharge (ECHO) (Table 2). There was a statistically

Table 1. Demographics, symptoms, comorbidities, and risk factors.

\begin{tabular}{|c|c|}
\hline & $\mathrm{n}$ \\
\hline Age (mean $\pm S D)$ & $53.5 \pm 20.4$ \\
\hline Male/Female, n & $11 / 11$ \\
\hline Symptoms & $\mathrm{n}(\%)$ \\
\hline Shortness of breath & $22(100)$ \\
\hline Central chest and flank pain & $18(81.8)$ \\
\hline Palpitation & $16(72.7)$ \\
\hline Cough & $15(68.2)$ \\
\hline Pain, redness, swelling in the leg & $13(59.1)$ \\
\hline Wheezing & $9(40.9)$ \\
\hline Producing sputum & $2(9.1)$ \\
\hline Hemoptysis & $1(4.5)$ \\
\hline Concomitant diseases and risk factors & $\mathrm{n}(\%)$ \\
\hline Immobilization & $12(54.5)$ \\
\hline Hypertension & $12(54.5)$ \\
\hline Deep vein thrombosis & $10(45.5)$ \\
\hline Orthopedic surgery & $6(27.3)$ \\
\hline Heart failure & $6(27)$ \\
\hline Diabetes & $5(22.7)$ \\
\hline Obesity & $5(22.7)$ \\
\hline Cesarean section & $4(18.2)$ \\
\hline Neurosurgery operation & $1(4.5)$ \\
\hline Lymphoma & $1(4.5)$ \\
\hline COVID-19 & $1(4.5)$ \\
\hline Breast carcinoma & $1(4.5)$ \\
\hline Cerebrovascular disease & $1(4.5)$ \\
\hline
\end{tabular}

SD: standard deviation.

Table 2. Mean value of pulmonary artery pressure at 1-year follow-up of patients.

\begin{tabular}{l|c}
\hline ECHO & PAP $($ mean \pm SD $)$ \\
\hline At hospitalization & $53.0 \pm 13.6$ \\
\hline At discharge from hospital & $42.7 \pm 13.4$ \\
\hline At month 1 & $37.6 \pm 12.6$ \\
\hline At month 3 & $30.2 \pm 13.1$ \\
\hline At month 6 & $25.4 \pm 10.1$ \\
\hline At the end of 1 year & $23.8 \pm 9.7$ \\
\hline
\end{tabular}

ECHO:Echocardiography; PAP:pulmonary artery pressure; SD:standard deviation. significant decrease in D-dimer and PAP levels before and after treatment. Notably, 19 patients with D-dimer levels below $0.5 \mathrm{ng} / \mathrm{mL}$ had PAP levels of $20 \mathrm{mmHg}$ and below at the end of treatment. At the end of 1 year, PAP was considered high in three patients. It was determined that three patients underwent $\mathrm{V} / \mathrm{P}$ scintigraphy and were evaluated in favor of chronic thromboembolic PHT, and one patient underwent endarterectomy (Figure 1).

\section{DISCUSSION}

When PTE is diagnosed, anticoagulant therapy should be started as soon as possible unless contraindications exist. In our study, the long-term outcomes of 22 patients diagnosed with submassive PTE and treated with UFH infusion were evaluated; accordingly, their PAP and D-dimer levels decreased significantly during follow-up, and PHT developed in three patients due to chronic PTE (CPTE).

Treatment of submassive PTE may be determined depending on the patient's clinical condition, drug contraindications, comorbidities, and hemodynamic findings. Although the role of systemic thrombolytic therapy is controversial, patients with clinical deterioration of submassive PTE are potential candidates for thrombolytic therapy ${ }^{6}$. A double-blind, randomized trial showed lower mortality with alteplase than with heparin alone in recurrent PTE, without the added risk of bleeding ${ }^{7}$. Another study comparing tenecteplase and heparin and examining 1,006 patients with submassive PTE reported less hemodynamic instability and mortality in the tenecteplase group ${ }^{8}$. The study by Rehman et al., which examined 86 patients with submassive pulmonary embolism, compared patients who received a

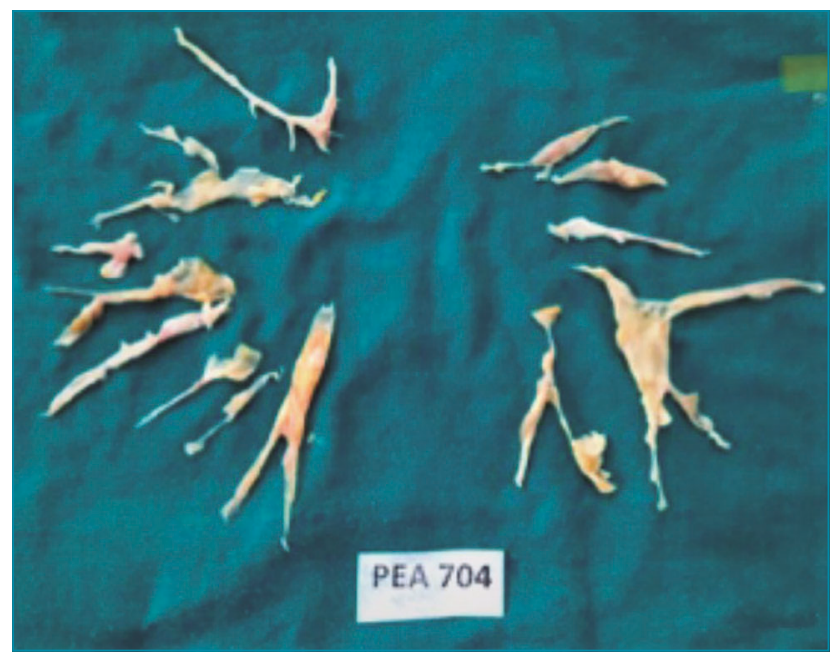

Figure 1. Endarterectomy tissues removed from pulmonary artery branches. 
thrombolytic followed by a heparin infusion with those who received a heparin infusion alone and concluded that the PAP scores of patients who received an early thrombolytic followed by a heparin infusion decreased significantly ${ }^{9}$. Three-year follow-up data from the PEITHO trial showed no difference in long-term mortality or incidence of chronic thromboembolic PHT between the thrombolytic and heparin infusion groups ${ }^{8}$.

The incidence of chronic thromboembolic PHT is $0.57 \%$ in the general population and $1.5 \%$ in patients with idiopathic $\mathrm{PTE}^{1}$. Factors predisposing to the development of PHT include recurrent venous thromboembolism and PTE of unknown cause. In our one-year follow-up, no recurrent PTE was detected. Thus, there was no case in which we could not detect the underlying risk factor. Nevertheless, $13.6 \%$ of chronic PHT were detected. PAP regressed significantly at one-year follow-up, and although the mean PAP of patients fell below $25 \mathrm{mmHg}$, this suggests that treatment with UFH does not prevent PHT development due to CPTE in submassive PTE. The main limitation of our study is the small number of patients and its descriptive study design. Studies on which treatment option prevents the development of CPTE in submassive PTE can be investigated with further case-control studies using larger patient collectives. Our descriptive study hypothesizes that UFH does not prevent the development of CPTE.

In a meta-analysis of 1,775 patients by Chatterjee et al., thrombolytic therapy was shown to be beneficial in reducing mortality, although it increased the risk of major bleeding $(9.24 \%)$ and intracranial hemorrhage (ICH) $(1.46 \%)^{4}$. Similarly, another meta-analysis by Riera-Mestre et al., which reviewed the outcomes of 1,833 patients, found that thrombolytic therapy reduced mortality despite the increased risk of major bleeding $(5.9 \%)$ and $\mathrm{ICH}(1.74 \%)^{5}$. Although studies on the use of thrombolytics in the treatment of submassive PTE have been accelerated, there are few studies investigating the use of UFH, which reduces the risk of bleeding and has been used safely for many years, in submassive PTE and its longterm outcomes. In our study, none of the patients developed major bleeding or ICH.

Heparin-induced thrombocytopenia is a complication of $\mathrm{PTE}^{10}$. In our study, thrombocytopenia was not found in any of the cases. However, when comparing the initial PAP and $\mathrm{PAP}$ at the end of one year, we determined that the values decreased significantly in our cases. PAP was $20 \mathrm{mmHg}$ or less in all, except in three patients. Although one case underwent endarterectomy for CPTE-related PHT, we did not detect any losses in our short- and long-term follow-up.

Post-PTE syndrome ${ }^{11}$, which is defined in the literature as a long-term complication that causes functional losses after an acute PTE episode, including a decrease in the patient's quality of life during long-term follow-up, was not studied in our patients. This is another limitation of our study.

\section{CONCLUSIONS}

Our study suggests that UFH is safe in the treatment of submassive PTE in terms of bleeding risk and reduces PAP, but its impact on PHT development due to CPTE during longterm follow-up needs to be investigated in further case-control studies with larger patient populations.

\section{AUTHORS' CONTRIBUTIONS}

LÖ: Conceptualization, Data curation, Formal Analysis, Writing - original draft, Writing - review \& editing. BÖ: Conceptualization, Formal Analysis, Writing - original draft, Writing - review \& editing. BA: Formal Analysis.

\section{REFERENCES}

1. Konstantinides SV, Meyer G, Becattini C, Bueno H, Geersing GJ, Harjola VP, et al. 2019 ESC Guidelines for the diagnosis and management of acute pulmonary embolism developed in collaboration with the European Respiratory Society (ERS). Eur Heart J. 2020;41(4):543-603. https://doi.org/10.1093/ eurheartj/ehz405

2. Chen LY, Ying KJ, Hong WJ, Zhou P. Comparison of low-molecular-weight-heparin and unfractionated heparin for acute PTE. J Zhejiang Univ Sci B. 2005;6(12):1195-9. https://doi.org/10.1631/jzus.2005.B1195

3. Martin C, Sobolewski K, Bridgeman P, Boutsikaris D. Systemic thrombolysis for pulmonary embolism: a review. P T. 2016;41(12):770-5. PMID: 27990080
4. Chatterjee S, Weinberg I, Yeh RW, Chakraborty A, Sardar P, Weinberg MD, et al. Risk factors for intracranial haemorrhage in patients with pulmonary embolism treated with thrombolytic therapy development of the PE-CH Score. Thromb Haemost. 2017;117(2):246-51. https://doi.org/10.1160/TH16-07-0588

5. Riera-Mestre A, Becattini C, Giustozzi M, Agnelli G. Thrombolysis in hemodynamically stable patients with acute pulmonary embolism: a meta-analysis. Thromb Res. 2014;134(6):126571. https://doi.org/10.1016/j.thromres.2014.10.004

6. Kearon C, AkI EA, Ornelas J, Blaivas A, Jimenez D, Bounameaux $\mathrm{H}$, et al. Antithrombotic therapy for VTE disease: CHEST guideline and expert panel report. Chest. 2016;149(2):31552. https://doi.org/10.1016/j.chest.2015.11.026 
7. Konstantinides S, Geibel A, Heusel G, Heinrich F, Kasper W, Management Strategies and Prognosis of Pulmonary Embolism-3 Trial Investigators. Heparin plus alteplase compared with heparin alone in patients with submassive pulmonary embolism. N Engl J Med. 2002;347(15):1143-50. https://doi. org/10.1056/NEJMoa021274

8. Steering Committee. Single-bolus tenecteplase plus heparin compared with heparin alone for normotensive patients with acute pulmonary embolism who have evidence of right ventricular dysfunction and myocardial injury: rationale and design of the Pulmonary Embolism Thrombolysis (PEITHO) trial. Am Heart J. 2012;163(1):33-38.e1. https://doi.org/10.1016/..ahj.2011.10.003
9. Rehman NU, Dar MI, Bansal M, Kasliwal RR. Clinical outcomes of submassive pulmonary embolism thrombolysis-an Indian experience. Egypt Heart J. 2020;72(1):87. https://doi. org/10.1186/s43044-020-00123-8

10. Bircan HA, Alanoglu EG. Massive pulmonary embolism in a patient with heparin induced thrombocytopenia: successful treatment with dabigatran. Eurasian J Med. 2016;48(1):65-8. https://doi.org/10.5152/eurasianjmed.2015.95

11. Piazza G. Advanced management of intermediate- and highrisk pulmonary embolism: JACC focus seminar. J Am Coll Cardiol. 2020;76(18):2117-27. https://doi.org/10.1016/j. jacc.2020.05.028 